\title{
ON THE EXTENSIONS TO THE BIDUAL OF A MAXIMAL MONOTONE OPERATOR
}

\author{
JEAN-PIERRE GOSSEZ
}

\begin{abstract}
An example is given which shows that a maximal monotone operator from a Banach space $X$ to its dual $X^{*}$ may have several extensions into a maximal monotone operator from $X^{* *}$ to $X^{*}$.
\end{abstract}

Introduction. Let $X$ be a real Banach space with dual $X^{*}$ and let $T: X$ $\rightarrow 2^{X^{*}}$ be a maximal monotone operator. Identifying as usual $X$ to a subspace of $X^{* *}$, we look at $T$ as a monotone operator from $X^{* *}$ to $2^{X^{*}}$; by Zorn's lemma, this operator can be extended into a maximal monotone operator from $X^{* *}$ to $2^{X^{*}}$. We are interested here in the question whether this extension is unique.

There are a number of cases where it is so.

Denote by $\bar{T}: X^{* *} \rightarrow 2^{X^{*}}$ the (monotone) operator whose graph is the closure of the graph of $T$ with respect to the weakest topology on $X^{* *} \times X^{*}$ which is stronger than $\sigma\left(X^{* *}, X^{*}\right) \times \sigma\left(X^{*}, X^{* *}\right)$ and such that $\left(x^{* *}, x^{*}\right)$ $\rightarrow\left\langle x^{* *}, x^{*}\right\rangle$ is upper semicontinuous. Since any maximal monotone extension of $T$ to $X^{* *}$ contains $\bar{T}$, we see that if $\bar{T}$ is maximal monotone, then $T$ has a unique maximal monotone extension to $X^{* *}$. An operator $T$ such that $\bar{T}$ is maximal monotone is called of dense type (a terminology slightly different from that of [2]). This kind of condition arises in the study of monotone operators in nonreflexive Banach spaces (cf. [2], [1], [7]). It is known, for instance, that the subdifferential of a convex function or the monotone operator associated with a saddle function are of dense type (cf. [6], [2], [5], [4]).

On the other hand, there are maximal monotone operators which are not of dense type but which have a unique maximal monotone extension to the bidual (cf. the example in [3]: the uniqueness assertion is contained in Proposition 1 of [3] and the fact that the operator considered there is not of dense type follows easily from relation (1) of [3]).

It is our purpose in this note to construct a maximal monotone operator which admits several (actually an infinity) maximal monotone extensions to the bidual. Our construction is based on a refinement of the method used in [3].

Received by the editors February 5, 1976.

AMS (MOS) subject classifications (1970). Primary 47H05; Secondary 46B10, 35J60. 
Example. Let $A: l^{1} \rightarrow l^{\infty}$ be the bounded linear operator defined by

$$
(A x)(n)=\sum_{m=1}^{\infty} x(m) K(m, n)
$$

for $x=(x(1), x(2), \ldots) \in l^{1}$, where the infinite matrix $K$ is constructed in the following way: take a bounded sequence $\left(r_{1}, r_{2}, \ldots\right)$ of real numbers in which each number $-n^{-2}(n=1,2, \ldots)$ appears infinitely many times and write

$$
K=\left[\begin{array}{ccccc}
0 & r_{1} & r_{2} & r_{3} & \cdots \\
-r_{1} & 0 & r_{2} & r_{3} & \cdots \\
-r_{2} & -r_{2} & 0 & r_{3} & \cdots \\
-r_{3} & -r_{3} & -r_{3} & 0 & \cdots \\
\cdot & \cdot & \cdot & \cdot & \\
\cdot & \cdot & . & \cdot & \\
\cdot & . & . & . & .
\end{array}\right] .
$$

The corresponding operator $A$ is then antisymmetric (i.e. $A \subset-A^{*}$ ) and thus (maximal) monotone.

PROPOSITION. There are infinitely many maximal monotone operators $B:\left(l^{\infty}\right)^{*}$ $\rightarrow 2^{10}$ which extend $A$.

Let us say that two points $\left(x^{* *}, x^{*}\right)$ and $\left(y^{* *}, y^{*}\right)$ in $X^{* *} \times X^{*}$ are monotonely related if $\left\langle x^{* *}-y^{* *}, x^{*}-y^{*}\right\rangle \geqslant 0$ and that a point $\left(x^{* *}, x^{*}\right)$ is monotonely related to a subset of $X^{* *} \times X^{*}$ if it is monotonely related to each point of this subset. Clearly, by Zorn's lemma, the proposition will be proved if we exhibit an infinite number of points in $\left(l^{\infty}\right)^{*} \times l^{\infty}$ such that each of them is monotonely related to the graph of $A$ but any two of them are not monotonely related.

LEMMA 1 (cf. [3]). Let $A: X \rightarrow X^{*}$ be a bounded linear antisymmetric operator. Then $\left(x^{* *}, x^{*}\right)$ is monotonely related to the graph of $A$ if and only if $x^{*}$ $=-A^{*} x^{* *}$ and $\left\langle x^{* *}, x^{*}\right\rangle \geqslant 0$.

Proof. Let $\left(x^{* *}, x^{*}\right)$ verify $\left\langle x^{* *}-y, x^{*}-A y\right\rangle \geqslant 0$ for all $y \in X$. Then

$$
\left\langle x^{* *}, x^{*}\right\rangle \geqslant\left\langle y, x^{*}\right\rangle+\left\langle x^{* *}, A y\right\rangle
$$

for all $y \in X$, which implies $\left\langle x^{* *}, x^{*}\right\rangle \geqslant 0$ and $x^{*}=-A^{*} x^{* *}$. The converse implication is proved by direct calculation. Q.E.D.

Let $\beta \mathbf{N}$ denote the Stone-Cech compactification of $\mathbf{N}$; then $l^{\infty}$ can be identified to the space $C(\beta \mathbf{N})$ of the continuous real-valued functions on $\beta \mathbf{N}$ and $\left(l^{\infty}\right)^{*}$ to the space $\mathscr{T}(\beta \mathbf{N})$ of the Radon measures on $\beta \mathbf{N}$. Given a bounded infinite matrix $K$, we consider for $m \in \mathbf{N}$ the function $K(m, \cdot)$ on $\mathbf{N}$ and extend it continuously on $\beta \mathbf{N}$; let $K(m, a)$ denote the value of this extension at $a \in \beta \mathbf{N}$. Then we consider for $a \in \beta \mathbf{N}$ the function $K(\cdot, a)$ on $\mathbf{N}$ and extend it continuously on $\beta \mathbf{N}$; let $K(b, a)$ denote the value of this 
extension at $b \in \beta \mathbf{N}$. If $K$ is antisymmetric, then the extended matrix $K(b, a)$ verifies $K(m, a)=-K(a, m)$ for $a \in \beta \mathbf{N}$ and $m \in \mathbf{N}$, but is generally not antisymmetric on $\beta \mathbf{N} \times \beta \mathbf{N}$, as is illustrated by the following simple example (cf. [3]):

$$
K(m, n)=0 \text { if } m=n, \quad-1 \text { if } n>m,+1 \text { if } n<m ;
$$

this example also gives some feeling for formula (5) below.

We will assume below that

$$
\text { for any } a \in \beta \mathbf{N} \backslash \mathbf{N}, K(m, a) \text { converges as } m \rightarrow \infty \text {; }
$$

this means that for any $a \in \beta \mathbf{N} \backslash \mathbf{N}, K(b, a)$ as a function of $b$ is constant on $\beta \mathbf{N} \backslash \mathbf{N}$. This condition is satisfied by the matrices (2) and (3).

LEMMA 2. Let $A: l^{1} \rightarrow l^{\infty}$ be a bounded linear antisymmetric operator with an associated matrix $K$ satisfying (4). Then

$$
\left\langle\mu,-A^{*} \mu\right\rangle=-\mu(\beta \mathbf{N} \backslash \mathbf{N}) \cdot \int_{\beta \mathbf{N} \backslash \mathbf{N}} K(b, a) d \mu(a)
$$

for all $\mu \in M(\beta \mathbf{N})$, where $b$ in the right-hand side is arbitrary in $\beta \mathbf{N} \backslash \mathbf{N}$.

Proof. First we deduce from (1) that

$$
(A x)(a)=\sum_{m=1}^{\infty} x(m) K(m, a)
$$

for $x \in l^{1}$ and $a \in \beta \mathbf{N}$. Indeed, if $n_{i} \in \mathbf{N}$ is a generalized sequence converging to $a$, then $K\left(\cdot, n_{i}\right)$ remains bounded in $l^{\infty}$ and converges componentwise to $K(\cdot, a)$; consequently $K\left(\cdot, n_{i}\right)$ converges to $K(\cdot, a)$ in $l^{\infty}, \sigma\left(l^{\infty}, l^{1}\right)$, and (6) follows from (1). Now we have

$$
\left(A^{*} \mu\right)(m)=\int_{\beta \mathbf{N}} K(m, a) d \mu(a)
$$

for $\mu \in \Re(\beta \mathbf{N})$ and $m \in \mathbf{N}$. Indeed, for any $y \in l^{1}$,

$$
\left\langle y, A^{*} \mu\right\rangle=\langle\mu, A y\rangle=\int_{\beta \mathrm{N}}\left[\int_{\mathbf{N}} y(m) K(m, a) d \nu(m)\right] d \mu(a)
$$

where $\nu$ denotes the counting measure on $\mathbf{N}$; equality (7) then follows from Fubini's theorem. Under assumption (4), we have

$$
\left(A^{*} \mu\right)(b)=\int_{\beta \mathbf{N}} K(b, a) d \mu(a)
$$

for $\mu \in \mathfrak{R}(\beta \mathbf{N})$ and $b \in \beta \mathbf{N}$. Indeed (7) gives

$$
\left(A^{*} \mu\right)(m)=\int_{\mathbf{N}} K(m, a) d \mu(a)+\int_{\beta \mathbf{N} \backslash \mathbf{N}} K(m, a) d \mu(a)
$$

by an argument similar to the preceding one involving $\sigma\left(l^{\infty}, l^{1}\right)$, we can pass 
to the limit in the first integral, and by Lebesgue's theorem, using assumption (4), we can pass to the limit in the second integral. Finally, for $\mu \in \mathfrak{R}(\beta \mathbf{N})$, we have

$$
\left\langle\mu,-A^{*} \mu\right\rangle=-\int_{\beta \mathbf{N}}\left[\int_{\beta \mathbf{N}} K(b, a) d \mu(a)\right] d \mu(b)
$$

writing each integral in the right-hand side as the sum of an integral over $\mathbf{N}$ and an integral over $\beta \mathbf{N} \backslash \mathbf{N}$, using the antisymmetry of $K$ and Fubini's theorem, we obtain (5). Q.E.D.

Proof of The Proposition. From the choice of $K$ (cf. (2)), we can find a sequence $a_{n}$ in $\beta \mathbf{N} \backslash \mathbf{N}$ such that $K\left(b, a_{n}\right)=-n^{-2}$ for $b \in \beta \mathbf{N} \backslash \mathbf{N}$. (Recall that when $a \in \beta \mathbf{N} \backslash \mathbf{N}, K(b, a)$ as a function of $b$ is constant on $\beta \mathbf{N} \backslash \mathbf{N}$.) Let $\mu_{n}=n \delta_{a_{n}}$ where $\delta_{a_{n}}$ denotes the Dirac measure at the point $a_{n}$. Using Lemma 1, we see that each $\left(\mu_{n},-A^{*} \mu_{n}\right)$ is monotonely related to the graph of $A$ since, by Lemma 2 ,

$$
\left\langle\mu_{n},-A^{*} \mu_{n}\right\rangle=-n \cdot\left(-n^{-2}\right) \cdot n \geqslant 0 .
$$

But if $n \neq m$, then $\left(\mu_{n},-A^{*} \mu_{n}\right)$ and $\left(\mu_{m},-A^{*} \mu_{m}\right)$ are not monotonely related since, by Lemma 2 ,

$$
\begin{aligned}
\left\langle\mu_{n}-\mu_{m},-A^{*} \mu_{n}+A^{*} \mu_{m}\right\rangle & =\left\langle\mu_{n}-\mu_{m},-A^{*}\left(\mu_{n}-\mu_{m}\right)\right\rangle \\
& =-(n-m) \cdot\left(-n^{-2} \cdot n+m^{-2} \cdot m\right)<0,
\end{aligned}
$$

which concludes the proof. Q.E.D.

REMARK. In the situation of the above Proposition, there are infinitely many linear maximal monotone operators $B: D(B) \subset\left(l^{\infty}\right)^{*} \rightarrow l^{\infty}$ which extend $A$. This follows easily from our construction and from the following two simple facts: (a) in a dual pair $(E, F)$, let $T: D(T) \subset E \rightarrow F$ be a linear monotone operator and let $(e, f) \in E \times F$ be monotonely related to the graph of $T$, with $e \notin D(T)$; then the linear extension of $T$ to $\operatorname{vct}[D(T), e]$ :

$$
\tilde{T}(x+\lambda e)=T(x)+\lambda f \text { for } x \in D(T) \text { and } \lambda \in \mathbf{R}
$$

is still monotone; (b) let $T: D(T) \subset E \rightarrow F$ be a linear monotone operator with a $\sigma(E, F)$ dense domain; if $T$ is maximal among all linear monotone operators, then $T$ is maximal monotone.

\section{REFERENCES}

1. T. Donaldson, Inhomogeneous Orlicz-Sobolev spaces and nonlinear parabolic initial value problems, J. Differential Equations 16 (1974), 201-256. MR 52 \# 1284.

2. J.-P. Gossez, Opérateurs monotones non linéaires dans les espaces de Banach non réflexifs, J. Math. Anal. Appl. 34 (1971), 371-395. MR 47 \#2442.

3. - On the range of a coercive maximal monotone operator in a nonreflexive Banach space, Proc. Amer. Math. Soc. 35 (1972), 88-92. MR 45 \# 7544.

4. _ On the subdifferential of a saddle function, J. Functional Analysis 11 (1972), 220-230. MR 50 \#2908. 
5. R. T. Rockafellar, Monotone operators associated with saddle-functions and minimax problems, Nonlinear Functional Analysis (Proc. Sympos. Pure Math., vol. XVIII, part 1), Amer. Math. Soc., Providence, R.I., 1970, pp. 241-250. MR 44 \#3159.

6. - On the maximal monotonicity of subdifferential mappings, Pacific J. Math. 33 (1970), 209-216. MR 41 \# 7432.

7. E. Zini, Théorèmes d'existence d'équations et d'inéquations non linéaires, C. R. Acad. Sci. Paris, Sér. A 281 (1975), A207-A209.

Defartement de Mathématique, Université libre de Bruxelles, Campus de la Plaine, C.P. 214, 1050 BRUXelles, Belgium 